\title{
Fatores associados à percepção e atitude de consumidores de carne bovina com certificação de origem em Uberlândia, Minas Gerais
}

\author{
Marcos Aurélio Lopes ${ }^{*}$, Érica Mariz Maia², Fábio Raphael Pascoti Bruhn², Izadora Alvarenga Custódio , \\ Christiane Maria Barcellos Magalhães da Rocha ${ }^{2}$, Peter Bittencout Faria ${ }^{2}$
}

10.1590/0034-737X201764010005

\section{RESUMO}

O objetivo deste estudo foi verificar os fatores socioeconômicos relacionados com a decisão de compra de carne com certificação de origem, além de levantar os perfis de percepção e de atitude dos consumidores de carne bovina, em Uberlândia, MG. Foi realizada a descrição das variáveis e construído um modelo múltiplo Generalized Estimating Equations (GEE) de regressão logística, para testar as possíveis associações entre as características socioeconômicas dos consumidores e os principais atributos da carne que influenciam a decisão sobre sua compra. As informações foram levantadas por meio de entrevistas com 213 consumidores, de abril a maio de 2012. A presença do carimbo do serviço de inspeção federal (SIF) ou estadual é o atributo que mais influencia a decisão de compra dos consumidores. A maioria dos entrevistados já ouviu falar sobre rastreabilidade bovina. Dentre estes, a maior parte está disposta a pagar mais pela carne com certificação de origem. Apesar disso, muitos consideram que o aumento do preço da carne é uma desvantagem da rastreabilidade. Consumidores com maior grau de escolaridade e renda apresentaram melhor conhecimento sobre este tipo de certificação, sendo esses fatores os de grande influência sobre a aceitação dos consumidores em pagar mais caro pela carne bovina rastreada.

Palavras-chave: comercialização; rastreabilidade; segurança alimentar.

\begin{abstract}
Factors associated with perceptions and attitude of consumers of beef with certificate of origin in Uberlândia, Minas Gerais, Brazil

The aim of this study was to verify the socioeconomic factors related to the decision to purchase beef with certificate of origin, as well as raise the profile of perception and attitude of consumers of beef in Uberlândia/MG. We performed a description of the variables and built a Generalized Estimating Equations (GEE) model of logistic regression to identify possible associations between the socio-economic characteristics of the consumers and the main attributes of the beef that influence its purchase. The information was gathered through interviews with 213 consumers, from April to May, 2012. The presence of the seal from the Federal Inspection Service (SIF) or the State Inspection Service (SIE) is the attribute that has more influence on the consumers' decision to purchase beef. Most respondents had already heard about bovine traceability. Among these, most would be willing to pay more for beef with certificate of origin. However, the majority consider that there are disadvantages associated with traceability, especially in relation to the increase in the price. Consumers with higher education and income had more knowledge about this type of certification, and these factors are of great influence on the consumers' willingness to pay more for traced beef.
\end{abstract}

Key words: commercialization; traceability; food safety.

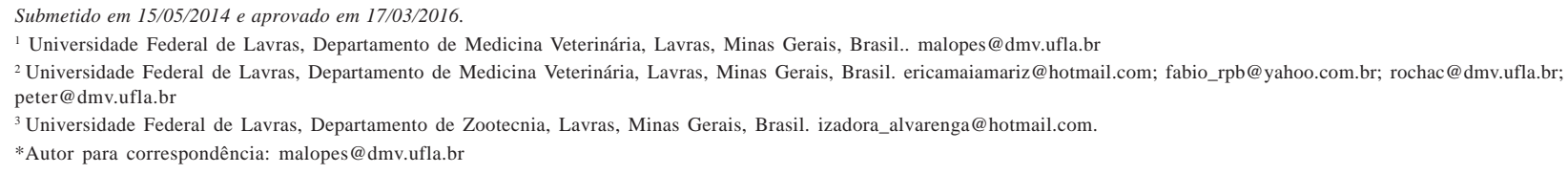




\section{INTRODUÇÃO}

A segurança sanitária dos alimentos desempenha um papel estratégico no comércio mundial e representa uma preocupação global (Nesbaken, 2009). De acordo com Sofos (2008), os desafios relacionados com a segurança de produtos de origem animal e as dificuldades no controle dos processos geram a necessidade de se criarem mecanismos que reduzam o risco potencial de doenças transmitidas por alimentos.

Neste sentido, destaca-se a rastreabilidade como meio de integração de toda a cadeia produtiva em um sistema de monitoramento e certificação, melhorando a percepção de segurança para os consumidores, graças à geração de maior quantidade de informações sobre a carne (Verbeke et al., 2010). Sendo o Brasil um dos maiores fornecedores de carne bovina para a União Europeia, uma das exigências para continuar com as exportações foi a implantação do programa brasileiro de rastreabilidade.

A rastreabilidade indica a possibilidade de seguir os passos de alguma coisa, no caso, o histórico do animal, desde o nascimento ou aquisição até o momento do seu consumo ou de uma de suas partes. Com ela é possível, através de uma etiqueta e um número vinculado a um corte da carne bovina, conhecer todo o manejo do animal, desde o seu nascimento até seu abate e comercialização (Lopes et al., 2007). Segundo Ubilava \& Foster (2009), a certificação de qualidade é uma segmentação de mercado que leva a maiores oportunidades para processadores de alimentos e varejistas, por serem considerados parte do sistema de gestão e envolver controle do sistema de produção.

Nesse contexto, os pontos de venda podem comercializar produtos classificados como carne rastreada ou, apenas, com certificação de origem. A carne rastreada difere da com certificação de origem (aquela cuja origem é atestada e reconhecida por uma certificadora), por apresentar outros dados importantes, como o registro de diversas ocorrências na vida do animal, como datas e tipos de vacinas, tratamentos veterinários associados, indicações do período de carência dos medicamentos administrados, transferências de propriedades e rotas de trânsito do animal, bem como sexo e aptidão (Lopes et al., 2007).

O sistema de rastreamentoe busca melhorar a segurança dos processos de obtenção da carne e dos seus produtos. Entretanto, estes benefícios não são tão claros para os consumidores, como são para os pesquisadores e para a indústria (Wezemael et al., 2011).

Torna-se relevante, portanto, avaliar se os consumidores conhecem o conceito de rastreabilidade bovina e se a carne com certificação de origem, que atualmente é comercializada nas redes de supermercados, está contemplada no desejo de compra, ainda que se pague mais caro por esse produto.
Por essa razão, os objetivos desta pesquisa foram: 1 . verificar a associação entre os atributos socioeconômicos dos consumidores e os principais atributos da carne que influenciam a decisão para sua compra; 2. avaliar o conhecimento sobre rastreabilidade bovina e a disposição para pagar a mais pela carne com certificação de origem e, 3 . levantar os perfis de percepção e de atitude dos consumidores de carne bovina rastreada, em Uberlândia, MG.

\section{MATERIAL E MÉTODOS}

Para avaliar a associação entre a percepção e a atitude de consumidores de carne bovina rastreada e algumas características sociodemográficas, foi realizado um estudo observacional de corte transversal (Medronho, 2008), em três supermercados de Uberlândia, cidade do Triângulo Mineiro, Minas Gerais, Brasil, de abril a maio de 2012. A definição da quantidade de entrevistados $(n=213)$ da amostra baseou-se em Barbetta (2007), considerando-se erro amostral máximo de 5\% e a população de 604.013 mil habitantes de Uberlândia (IBGE, 2010).

Visando a obter informações sobre a caracterização da percepção e da atitude de consumidores de carne bovina, foram realizadas entrevistas, a partir de formulário estruturado (36 perguntas fechadas) (Tabela 1), adaptados de Velho et al. (2009) e de Rigueira et al. (2014). Ainda, para todos os entrevistados foram obtidas informações sobre características sociodemográficas relacionadas com o sexo, idade (até 40 anos; mais de 40 anos), escolaridade (até segundo grau; graduação ou pós-graduação) e renda (até seis salários mínimos; mais que seis salários mínimos), utilizadas como variáveis independentes no estudo. No período da coleta das informações, o salário mínimo correspondia ao valor de $\mathrm{R} \$ 622,00$.

Os entrevistados foram selecionados aleatoriamente, sendo convidados enquanto se encontravam em frente à gôndola de carnes, nos três supermercados pesquisados. Para os consumidores que nunca haviam ouvido falar sobre carne bovina rastreada, a entrevista se restringia às questões relativas às características sociodemográficas.

Para identificar as características sociodemográficas associadas à percepção e à atitude dos consumidores em relação à carne bovina com certificação de origem, foi realizada a análise univariada por testes qui-quadrado $\left(x^{2}\right)$ ou Exato de Fischer. Pelo teste Exato de Fischer, quando havia menos que cinco observações no quadrante do teste. As variáveis que mostraram associação com $\mathrm{p} \leq 0,2$ pelo teste de $x^{2}$ ou pelo Exato de Fischer foram selecionadas para a construção do modelo múltiplo.

Assim, a existência de associação entre os fatores foi verificada, utilizando-se o modelo múltiplo Generalized Estimating Equations (GEE) de regressão logística, que é mais adequado quando se observam dados associados em diferentes dimensões (Hanley et al., 2003). Neste estu- 
do, foram consideradas as respostas de consumidores presentes em diferentes supermercados (considerados sujeitos do modelo). A análise GEE permite a avaliação de associação de múltiplos indivíduos, considerando-se, no modelo, o supermercado em que compravam, assumindo-se que os casos são dependentes, dentro de cada sujeito e independentes entre sujeitos. Para todas as variáveis presentes no modelo final ( $\mathrm{p}<0,05$ ), foi estimado o risco, por meio da Odds Ratio (OR) ajustada e com intervalo de confiança de $95 \%$.

\section{RESULTADOS E DISCUSSÃO}

As características sociodemográficas dos consumidores de carne de Uberlândia entrevistados podem ser observadas na Tabela 2. Entre aqueles que já ouviram falar em carne rastreada, os principais critérios considerados na decisão de compra da carne bovina foram a presença do carimbo do serviço de inspeção federal (SIF) ou estadual $(29,68 \%)$, seguida da presença do selo de qualidade $(18,71 \%)$, do preço e do estabelecimento comercial onde foi realizada a aquisição da carne (ambos com 14,20\%), o menor teor de gordura $(6,46 \%)$, o cheiro $(5,81 \%)$, a cor (3,88\%), a textura (2,59\%), o local onde o animal foi abatido e o preparo rápido e fácil da carne (ambos com 1,94\%). Além disso, 62,59\% citaram como conceito de carne rastreada o "produto que traz as informações de origem".

Nenhum entrevistado considerou que não há benefício no consumo da carne rastreada em relação ao da não rastreada. Os maiores benefícios considerados pelos consumidores foram a maior segurança alimentar e o menor risco para a saúde pública $(78,71 \%)$; o acesso à informa- ção referente a idade, sexo, raça e outras características do animal abatido (8,39\%). Grande parte dos entrevistados $(68,41 \%)$ considerou que há desvantagens da carne rastreada em relação à sem rastreamento, sendo o aumento do preço do produto a principal alegação (41,30\%). Apesar disso, a maior parte relatou que aceitaria pagar mais caro pela carne rastreada $(74,83 \%)$, mas somente com acréscimo de menos de 5\% (24,52\%).

Tabela 2: Características sociodemográficas dos consumidores de carne entrevistados ( $\mathrm{n}=213$ ), Uberlândia, MG, Brasil, 2012

\begin{tabular}{lcc}
\hline Variável & Categoria & $\%$ \\
\hline Sexo & Masculino & 60,1 \\
& Feminino & 39,9 \\
\hline Idade & $20-30$ anos & 21,13 \\
& $31-40$ anos & 26,29 \\
& $41-50$ anos & 23,47 \\
& $51-60$ anos & 21,60 \\
Escolaridade & Até $1^{\circ}$ grau & 7,51 \\
\hline 2 & grau & 8,45 \\
$3^{\circ}$ grau & 40,38 \\
$4^{\circ}$ grau & 33,33 \\
falar em & Até 3 salários mínimos & 17,84 \\
\hline 4-6 salários mínimos & 21,60 \\
& 7-10 salários mínimos & 34,20 \\
& Mais de 11 salários mínimos & 22,60 \\
& Não respondeu & 15,50 \\
& Não & 6,10 \\
\hline
\end{tabular}

*salário mínimo $=\mathrm{R} \$ 622,00$

Tabela 1: Resumo das características levantadas em entrevistas aplicadas a consumidores de carne bovina, em supermercados de Uberlândia, MG, Brasil, 2012

\begin{tabular}{ll}
\hline Item & Variáveis dicotômicas (Respostas: não/sim) \\
\hline \multirow{2}{*}{ Atitude } & Consumo de produtos de origem animal com certificação. \\
& Disposição para pagar mais caro pelo produto de origem animal certificado. \\
& Disposição para pagar a mais pela carne rastreada: 1 . Até $5 \% ; 2.5 \% ; 3.10 \% ; 4.15 \% ; 5.20 \% ;$ ou 6 mais que $20 \%$.
\end{tabular}

Conhecimento prévio sobre rastreabilidade, carne rastreada ou carne com garantia de origem.

Atributo mais importante na decisão de compra da carne: 1. presença do selo de qualidade; 2. carimbo do SIF;

3. preço; 4. quantidade de gordura; 5. preparo rápido/fácil; 6. cria ou engorda; 7. local de abate; 8. Local de compra;

9. raça; 10. sexo do animal; 11. Maciez/textura; 12. odor; 13. cor da carne.

Conceito de carne rastreada: 1 . Carne que traz as informações nutricionais na embalagem; 2 . Produto cárneo que não apresenta contaminantes ou resíduos químicos; 3. Carne que traz as informações relacionadas com o sistema de

Percepção produção e com a origem do animal; e 4. Carne com fiscalização pelo sistema de inspeção federal ou serviço estadual.

Benefícios em relação aos quais a carne rastreada poderia superar a sem rastreabilidade: 1 . maior competitividade no mercado exterior, 2. melhor qualidade, mais saborosa e nutritiva; 3. maior segurança e menor risco à saúde pública; 4. acesso a informações referentes à idade, sexo, raça e outras; e 5. Nenhum

Desvantagens que a carne rastreada poderia trazer: 1 . maior preço; 2. menor oferta; 3 . valorização de todas as carnes; e 4. Nenhuma. 
As atitudes e percepções de consumidores de carne bovina, em Uberlândia, estatisticamente associadas a sexo, idade, renda e escolaridade encontram-se expostas nas Tabelas 3, 4, 5 e 6, respectivamente. Assim, os entrevistados do sexo feminino e os com mais de 40 anos apresentaram menores possibilidades de já terem ouvido falar em carne rastreada, ao contrário dos entrevistados que citaram receber mais de seis salários mínimos e que possuem graduação, que tiveram mais oportunidades de conhecimentos sobre carne com rastreabilidade.

Os consumidores com mais de 40 anos mencionaram o carimbo do SIF como atributo mais importante na compra da carne com rastreabilidade. Por outro lado, os entrevistados que alegaram ter renda mensal menor que seis salários mínimos consideraram o preço como atributo mais importante. Os indivíduos com graduação consideraram que a baixa quantidade de gordura fosse o atributo mais importante no momento da compra da carne rastreada.

Dentre os entrevistados que tiveram conhecimento sobre carne rastreada, os graduados mostraram menores possibilidades de definir o conceito de carne rastreada como "o produto cárneo que não possui contaminantes ou resíduos químicos". Ainda, os que possuíam escolaridade superior mencionaram que a carne rastreada é a "carne que traz as informações relacionadas ao sistema de produção e à origem do animal".

Segundo a pesquisa, as consumidoras mostraram menor chance de considerar, como benefício da carne rastreada, a maior competitividade no mercado exterior. Ainda, as mulheres mostraram-se mais aptas a considerar que a carne rastreada supera a não rastreada em oferecer mais segurança e menor risco de doenças transmitidas por alimento para a população.
Dos entrevistados, aqueles com ensino superior completo apresentaram menor possibilidade de considerar a valorização de todas as carnes como desvantagens que a carne com certificação de rastreabilidade poderia trazer. Também em relação ao aumento do valor da carne rastreada, a pesquisa mostrou que os indivíduos com nivel de escolaridade superior mostraram menores possibilidades de aceitarem pagar um valor menor que 5\% a mais pela carne com rastreabilidade. Ainda, os entrevistados graduados mostraram maiores disponibilidades a pagar $10 \%$ a mais pela carne rastreada.

Alguns autores citaram que o consumidor realiza a sua decisão de compra da carne com base em grande quantidade de informações (preço, rótulo, marca, aparência e tipo de corte), que, por sua vez, estariam relacionados, na sua concepção de qualidade, com os atributos maciez, sabor, frescor e nutrição (Krystallis et al., 2007; Verbeke et al., 2010; Troy \& Kerry, 2010). Contudo, esse comportamento está mudando e os consumidores têm voltado maior atenção e preocupação em relação às caracteriticas extrínsecas dos produtos, ou seja, fatores relacionados com o sistema de produção animal, como a utilização de outras tecnologias (irradiação de alimentos, produção orgânica, técnicas de biotecnologia, uso de antibióticos, de pesticidas e de hormônios de crescimento) (Unnevehr et al., 2010). Segundo Sato \& Silva (2008), o perfil do consumidor também está mudando, graças ao maior acesso à informação, e estão cada vez mais exigentes em relação aos atributos de qualidade da carne.

Por exemplo, em um levantamento com consumidores de carne bovina de cinco países europeus (França, Alemanha, Polônia, Espanha e Reino Unido), Wezemael et al. (2011), verificaram que o maior nível de confiança e a percepção de risco para a carne bovina estavam associados a

Tabela 3: Atitudes e percepções de 213 consumidores de carne bovina, associadas ao gênero, Uberlândia, Minas Gerais, 2012

\begin{tabular}{|c|c|c|c|c|c|c|}
\hline Gênero & \multicolumn{3}{|c|}{ Questão levantada } & Valor de p & Odds Ratio & $\mathrm{IC}(95 \%)$ \\
\hline & \multicolumn{3}{|c|}{ Já ouviu falar sobre rastreabilidade, carne rastreada } & & & \\
\hline & \multicolumn{3}{|c|}{ ou carne com garantia de origem? } & & & \\
\hline & Não & Sim & Total & & & \\
\hline Masculino & 22 & 105 & $127(82,7 \%)$ & 0,001 & 1 & $0,216-0,384$ \\
\hline \multirow[t]{4}{*}{ Feminino } & 35 & 50 & $85(58,2 \%)$ & & 0,288 & \\
\hline & \multirow{2}{*}{\multicolumn{3}{|c|}{$\begin{array}{l}\text { Maior competitividade da carne brasileira no } \\
\text { mercado exterior como vantagem da carne rastreada. }\end{array}$}} & & & \\
\hline & & & & & & \\
\hline & Não & Sim & Total & & & \\
\hline Masculino & 94 & 11 & $105(10 \%)$ & 0,007 & 1 & $0,049-0,619$ \\
\hline \multirow[t]{4}{*}{ Feminino } & 49 & 1 & $50(2 \%)$ & & 0,174 & \\
\hline & \multirow{2}{*}{\multicolumn{3}{|c|}{$\begin{array}{l}\text { Oferecer mais segurança alimentar } \\
\text { como vantagem da carne rastreada. }\end{array}$}} & & & \\
\hline & & & & & & \\
\hline & Não & Sim & Total & & & \\
\hline Masculino & 28 & 77 & $105(73 \%)$ & 0,001 & 1 & $1,691-6,333$ \\
\hline Feminino & 5 & 45 & $50(90 \%)$ & & 3,273 & \\
\hline
\end{tabular}

IC (95\%): intervalo de confiança a 95\%

Rev. Ceres, Viçosa, v. 64, n.1, p. 031-039, jan/fev, 2017 
uma melhor aceitação dos consumidores para o uso de técnicas que melhorassem a sua segurança, principalmente em relação ao seu processo de obtenção, como no caso da adoção da inspeção do produto e da rastreabilidade no sistema produtivo.

Neste estudo, o preço da carne foi citado como fator mais importante na decisão de compra por apenas 14,20\% dos entrevistados, sendo o terceiro fator na ordem de citação, junto com o estabelecimento comercial onde foi realizada a aquisição da carne. Resultado semelhante foi encontrado por Tonsor \& Marsh (2007), nos Estados Unidos, ao verificarem que $75 \%$ da variabilidade da demanda ou de consumo está relacionada com os fatores preço da carne e renda do consumidor. Porém, segundo Verbeke \& Vackier (2004) e Hocquette et al., (2012), o atributo preço é geralmente o fator considerado de maior impacto na decisão da compra da carne pelo consumidor. Angulo \& Gil (2007), em estudo realizado na Espanha, relataram que o preço é um dos fatores mais importantes na percepção do risco de segurança alimentar e que os consumidores acreditam que produtos mais caros apresentam algum tipo de vantagem. Krystallis et al. (2007), na Grécia, verificaram que os consumidores desse país preferem realizar a compra da carne diretamente em açougues, onde acreditam poder ter certeza de sua origem, em vez de consultarem as informações disponíveis nos rótulos dos produtos encontrados em supermercados. Ou seja, preferem confiar na qualidade visual, em vez de em marcas e certificações de qualidade.

A valorização da qualidade do produto, para as classes com renda mais baixa, perde em importância para o preço do produto. Neste estudo, a aparência (cor, textura) e o cheiro foram citados pela minoria dos entrevistados como fatores observados no momento da compra da carne bovina. Ao contrário do observado neste estudo, Velho et al. (2009), no Estado do Rio Grande do Sul, verificou-se que a aparência, principalmente relacionada com a cor da carne, foi o aspecto mais citado como importante na decisão de compra. Esse comportamento é relatado por diversos autores, uma vez que a cor é apontada como o índice de frescor e qualidade mais óbvia para o consumidor (Krystallis et al., 2007).

Brumm \& Terra (1988) salientaram que a carne mais escura ou com coloração esverdeada e superfície úmida pode sugerir estado de decomposição, com desenvolvimento de bactérias que aumentam o risco de doenças transmitidas por alimentos. Ubilava \& Foster (2009) sugeriram, por meio de um modelo de regressão logística que, na República da Georgia, tanto os consumidores quanto os integrantes da cadeia produtiva da carne deviam preocuparse primeiramente com a aparência visual da carne, a fim de se evitar a compra de produtos estragadados.

Neste estudo, a maior parte $(62,59 \%)$ dos entrevistados citou como conceito de carne rastreada "aquela que traz as informações de origem". Os resultados sobre o conhecimento a respeito de rastreabilidade, verificados nesta pesquisa, foram maiores que os encontrados por Zhao et al. (2010) para os quais apenas 3\% dos 588 entrevistados, nas cidades de Beijing e Xianyang, na China, estavam familiarizados com o real conceito de carne rastreada. Um dos fatores que contribuiu para o baixo índice de familiaridade, na China, segundo esses autores, é o fato de que a rastreabilidade é recente naquele país, sendo as primeiras importações realizadas em 2009.

Nenhum entrevistado considerou que não há benefício da carne rastreada, em comparação com a não rastreada. Mørkbak et al. (2008) apontaram que os consumidores apresentam uma disposição positiva à compra do produ-

Tabela 4: Atitudes e percepções de 213 consumidores de carne bovina, associadas à idade, Uberlândia, Minas Gerais, 2012

\begin{tabular}{|c|c|c|c|c|c|c|}
\hline Idade & \multicolumn{3}{|c|}{ Questão levantada } & Valor de $p$ & Odds Ratio & IC $(95 \%)$ \\
\hline \multicolumn{7}{|c|}{ Já ouviu falar sobre rastreabilidade, } \\
\hline & Não & $\operatorname{Sim}$ & Total & & & \\
\hline Até 40 anos & 23 & 78 & $101(77 \%)$ & 0,001 & 1 & $0,522-0,752$ \\
\hline \multirow[t]{3}{*}{ Mais que 40 anos } & 34 & 77 & $111(69 \%)$ & & 0,627 & \\
\hline & \multicolumn{3}{|c|}{$\begin{array}{l}\text { Carimbo do SIF como fator mais importante } \\
\text { na tomada de decisão de compra da carne. }\end{array}$} & & & \\
\hline & Não & Sim & Total & & & \\
\hline Até 40 anos & 61 & 17 & $78(22 \%)$ & 0,007 & 1 & $1,235-3,806$ \\
\hline \multirow[t]{3}{*}{ Mais que 40 anos } & 48 & 29 & $77(38 \%)$ & & 2,168 & \\
\hline & \multicolumn{3}{|c|}{ Preço como fator mais importante } & & & \\
\hline & Não & Sim & Total & & & \\
\hline Até 40 anos & 63 & 15 & $78(19 \%)$ & 0,010 & 1 & $0,241-0,821$ \\
\hline Mais que 40 anos & 70 & 7 & $77(9 \%)$ & & 0,445 & \\
\hline
\end{tabular}

IC (95\%): intervalo de confiança a $95 \%$ 
to, quando, dentre outros atributos, existe a garantia de que ele é seguro. Segundo Grunert et al. (2004), é necessário assegurar uma garantia de origem do produto, mostrando que ele foi produzido dentro de boas práticas de fabricação e que está apto a receber seu certificado de origem, analogicamente ao certificado de garantia de qualquer outro produto, mostrando confiabilidade para a sua compra. Para aprimorar a percepção de segurança alimentar, Angulo \& Gil (2007) salientaram que os sistemas de controle da qualidade devem ser fortalecidos e divulgados para o maior conhecimento da população.

Grande parte dos entrevistados $(68,41 \%)$ considerou que há desvantagens da carne rastreada em relação à sem rastreamento, sendo a principal alegação o aumento do preço do produto. Mesmo assim, a maior parte $(74,83 \%)$ relatou que aceitaria pagar mais caro pela carne rastreada, mas somente um acréscimo de até $5 \%(24,52 \%)$, o que pode ser considerado um valor baixo. De acordo com Lazzarotto (2011), no Brasil a carne certificada pode apresentar valores até $30 \%$ maiores do que os do produto que não apresenta certificação de origem. Zhao et al. (2010) encontraram percentuais superiores aos encontrados neste estudo, relatando que a maioria dos entrevistados na China pagaria de 9 a $12 \%$ a mais por produtos rastreados.

$\mathrm{Na}$ Coréia do Sul, onde grande parte da carne consumida é importada, Lee et al. (2011) verificaram disposição dos consumidores de pagarem até $39 \%$ para a carne bovina rastreada, em comparação com a não rastreada. Esse resultado reflete a preocupação dos consumidores com a segurança alimentar, em relação à carne importada dos Estados Unidos, por causa da ocorrência da BSE (Encefalopatia Espongiforme Bovina), em anos anteriores.
Os custos com a rastreabilidade, segundo alguns autores, são suportáveis pela indústria, em decorrência de uma maior demanda do produto, graças ao aumento da confiança dos consumidores em relação à carne bovina (Resende Filho, 2008; Pendell et al., 2010). Apesar disso, os resultados deste trabalho mostraram, ainda, a existência de menores sensibilidade e percepção dos consumidores brasileiros, quanto aos reais benefícios do sistema, em vista do baixo valor que estão dispostos a pagar para produtos rastreados. Esse comportamento pode ser devido ao fato da não ocorrência de casos da doença BSE e à posição do país como grande exportador de carne. Resende Filho (2008), avaliando potenciais benefícios da rastreabilidade animal para o setor de carnes americano, estimou que o custo para a implantação do sistema seria pago com o ganho em receita, com uma estimativa entre o custo e a receita em torno de $30 \%$. Pendell et al. (2010) realizaram, nos Estados Unidos, uma avaliação do impacto sobre o custo de produção do uso da identificação e rastreamento, citando que representariam os seguintes percentuais de aumento: valor de $0,007 \%$ do preço da carne no atacado; $0,12 \%$ no abate de bovinos e, de $0,43 \%$ na fazenda.

Neste estudo, a escolaridade foi o fator de maior influência sobre a percepção e a atitude dos entrevistados que já tinham ouvido falar em rastreabilidade bovina. Foi verificado que indivíduos com maior escolaridade tendem a apresentar melhor percepção sobre a rastreabilidade e o correto conceito de carne rastreada, que se refletem em melhor atitude, relacionada com a disposição a pagar mais pela carne rastreada, e também a se preocuparem menos com o aumento do preço do produto rastreado, em comparação com o do não rastreado. Esses

Tabela 5: Atitudes e percepções de 213 consumidores de carne bovina, associadas à renda, Uberlândia, Minas Gerais, 2012

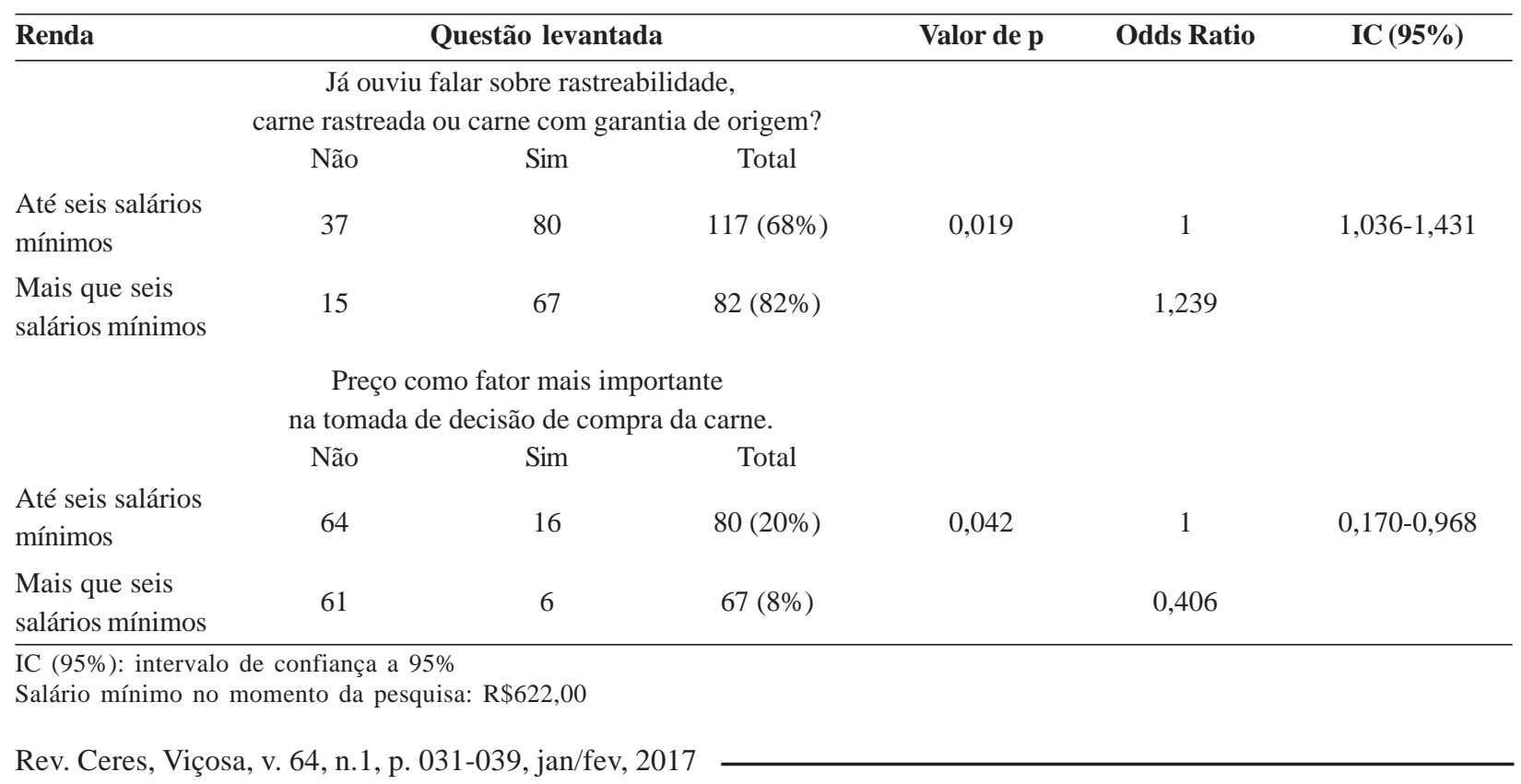


resultados indicaram que a escolaridade melhora a consciência das pessoas em relação à importância da carne rastreada, graças ao maior acesso às informações sobre sua obtenção, proporcionando maior transparência e compreensão das etapas envolvidas no processo de produção, o que acarreta maior nível de garantia e segurança ao produto final. Neste estudo, também foi observado que indivíduos com maior renda mensal e com mais idade apresentam maior probabilidade de aceitar pagar mais caro pela carne com certificação de origem. É provável que isso esteja relacionado com a percepção correta da carne rastreada, relatada por este grupo de indivíduos, indicando que o conhecimento sobre o conceito correto e os benefícios da carne rastreada está relacionado com uma atitude favorável ao consumo desse produto. Ou seja, maiores níveis de escolaridade e de renda e maior idade são fatores de grande influência sobre a aceitação dos consumidores a quanto pagarem a mais pela carne bovina rastreada, como era esperado, o que provavelmente está associado à melhor percepção desses indivíduos dos benefícios deste tipo de produto. Comportamento semelhante foi verificado em outros países, indicando que este tipo de consumidor representa dois terços do mercado e corresponde à parcela das pessoas em que o marketing e a divulgação de informações sobre rastreabilidade promoveriam melhores resultados (Verbeke \& Vackier, 2004; Krystallis et al., 2007).

Neste estudo, maior idade também se mostrou associada à importância dada à presença de carimbos de inspeção federal (SIF) ou estadual. Por outro lado, na Grécia,

Tabela 6: Atitudes e percepções de 213 consumidores de carne bovina, associadas à escolaridade, Uberlândia, Minas Gerais, 2012

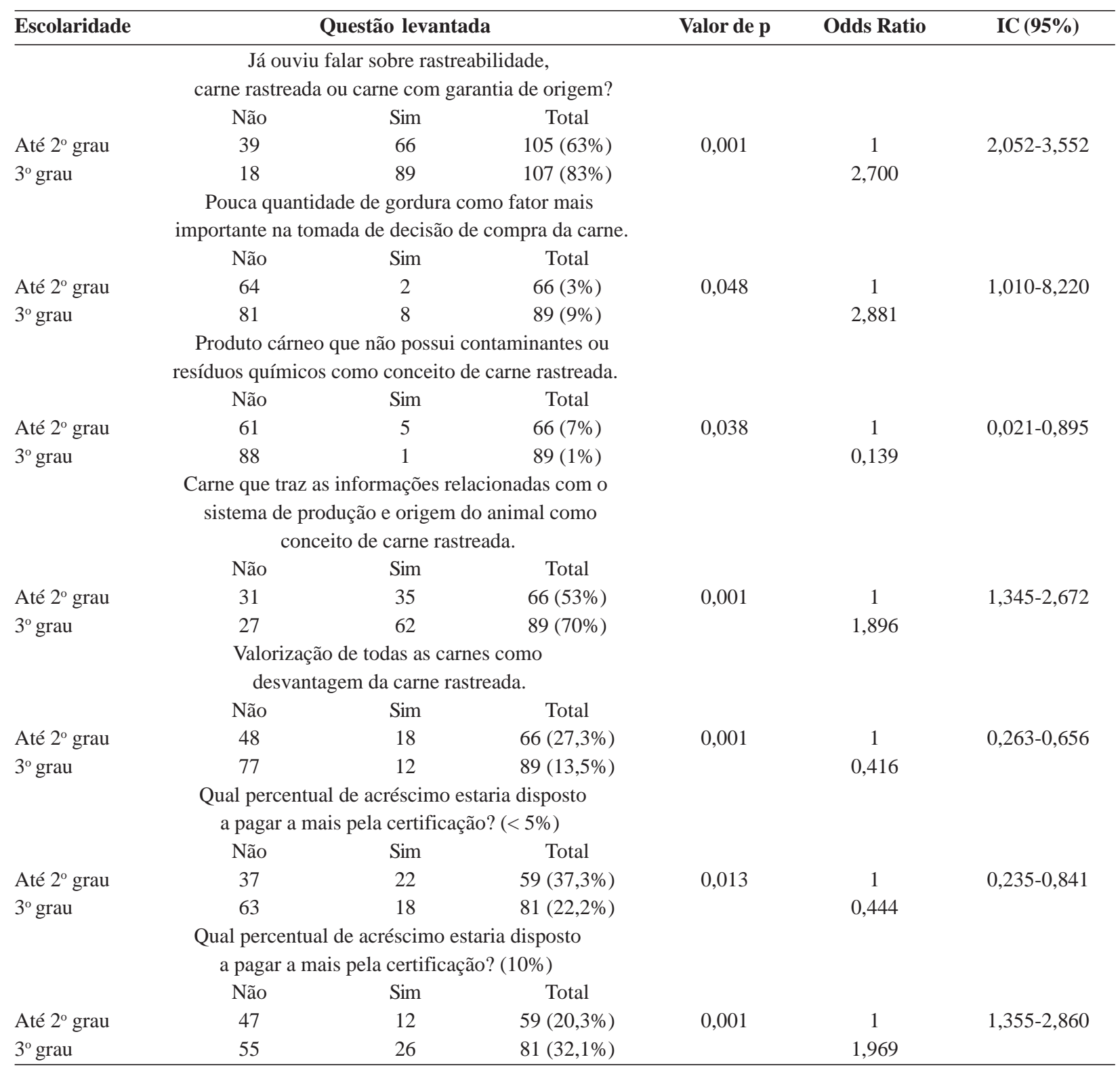

IC (95\%): intervalo de confiança a $95 \%$ 
os entrevistados com idade acima de 60 anos consideram a qualidade visual do produto como o atributo mais importante, enquanto os de meia idade (entre 40 e 60 anos) levam em consideração tanto os aspectos sensoriais quanto os de segurança alimentar. Entretanto, na Bélgica e Grécia, pessoas mais jovens (abaixo de 25 e 40 anos, respectivamente) seriam mais indiferentes aos aspectos sensoriais da carne, mostrando menor percepção dos atributos relacionados com a qualidade e segurança, em virtude do seu menor envolvimento nos processos de compra e consumo (Verbeke \& Vackier, 2004; Krystallis et al., 2007).

Outro aspecto, que reflete o efeito positivo do aumento do conhecimento sobre a rastreabilidade são os consumidores de carne que têm filhos, fator não pesquisado neste trabalho. Segundo Verbeke \& Vackier (2004), esse público demonstra maior cautela na tomada de decisão no momento da compra de carne, buscando informações a respeito da origem e das características dos produtos, com maior preocupação em relação à segurança alimentar e a uma dieta saudável.

Considerando-se o perfil dos consumidores de carne bovina em seus diferentes aspectos, para proporcionar maior difusão e demanda para os produtos com certificação de origem ou rastreados, é necessária a adoção de estratégias de veiculação de informações de forma mais direta, principalmente a partir do local onde adquirem os produtos, como redes de supermercados e casas de carnes especializadas.

\section{CONCLUSÕES}

A maioria dos entrevistados já tem conhecimento sobre o termo "carne rastreada".

A certificação de qualidade do produto, como selo e carimbo do SIF, foi a maior preocupação dos consumidores, enquanto os fatores intrínsecos e extrínsecos apresentaram menor influência na tomada de decisão no momento da compra.

O sexo e idade influenciaram nas decisões de compra de carne.

O maior conhecimento sobre rastreabilidade, verificado para os consumidores com maior renda familiar e escolaridade, indicaram que esse público apresentou melhor percepção em relação aos aspectos de qualidade de carne e buscaram maiores informações a respeito dos produtos que estão adquirindo e, assim, mostraram maior disposição para pagar mais pela carne com certificação de origem.

\section{AGRADECIMENTOS}

Os autores agradecem ao CNPq pelo apoio recebido.

\section{REFERÊNCIAS}

Angulo AM \& Gil AJM Risk (2007) Perception and consumer willingness to pay for certified beef in Spain. Food Quality and Preference, 18:1106-1117.

Barbetta PA (2007) Estatística aplicada às Ciências Sociais. $7^{\text {a }}$ ed. Florianópolis, UFSC. 315p.

Brumm MAR \& Terra NM (1988) Carne e seus derivados: Técnicas de Controle de Qualidade. São Paulo, Nobel. 425p.

Grunert KG, Brunso K \& Bredahl L (2004) Review: Consumer perception of meat quality and implications for product development in the meat sector. Meat Science, 66:259-272.

Hanley AJ, Negassa A, Edwardes MDB \& Forrester JE (2003) Statistical Analysis of Correlated Data Using Generalized Estimating Equations: An Orientation. American Journal of Epidemiology, 157:364-375.

Hocquette JF, Botreau R, Picard B, Jacquet A, Pethick DW \& Scollan ND (2012) Opportunities for predicting and manipulating beef quality. Meat Science, 92:197-209.

IBGE - Instituto Brasileiro de Geografia e Estatística (2010) Cidades. Disponível em: <http://www.ibge.gov.br/cidadesat/ topwindow.htm?1>. Acessado em: 23 de fevereiro de 2012.

Krystallis A, Chryssochoidis G \& Scholderer J (2007) Consumerperceived quality in 'traditional' food chains: The case of the Greek meat supply chain. Appetite, 48:54-68.

Lazzarotto NF (2011) Estudos sobre o mercado de certificações em alimentos no Brasil. Disponível em: <http:// www.fearp.usp.br/egna/resumos/Lazzarotto.pdf> Acessado em: 21 de junho de 2011.

Lee JY, Han DB, Nayga Junior RM \& Lim SS (2011) Valuing traceability of imported beef in Korea: an experimental auction approach. The Australian Journal of Agricultural and Resource Economics, 55:360-373.

Lopes MA, Santos G, Amado GB \& Cardoso MG (2007) Principais dificuldades encontradas pelos pecuaristas na implantação da rastreabilidade de bovinos. Ciência Animal Brasileira, 8:515-520.

Medronho RA (2008) Epidemiologia. São Paulo, Atheneu. 790p.

Mørkbak MR, Christensen T \& Gyrd-Hansen D (2008) Valuation of food safety in meat e a review of stated preference studies. Food Economics, 5:63-74.

Nesbaken T (2009) Food Safety in a global market - Do we need to worry? Small Ruminant Research, 86:63-66.

Pendell DL, Brester GW, Schroeder TC, Dhuyvetter KC \& Tonsor GT (2010) Animal identification and tracing in The United States. American Journal Agricultural Economics, 92:927-940.

Resende Filho MA (2008) Potenciais benefícios do sistema de rastreabilidade animal dos EUA para o setor de carnes americano. Revista de Economia e Sociologia Rural, 46:1129-1154.

Rigueira LL, Lopes MA, Bruhn FRP, Rodrigues CG \& Faria PB (2014) Willingness of the consumers of the Federal District “ Brazil " to purchase beef meat with certification of origin. Arquivo Brasileiro de Medicina Veterinária e Zootecnia, 66:19461950.

Sato GS \& Silva ROP (2008) Fragilidade do modelo de rastreabilidade da carne bovina brasileira. Análises e Indicadores do Agronegócio, 3:1-5.

Sofos JN (2008) Challenges to meat safety in the $21^{\text {st }}$ century. Meat Science, 78:3-13.

Tonsor G \& Marsh GL (2007) Comparing Heterogeneous Consumption in U.S. and Japanese Meat Fish Demand. Agricultural Economics, 37:81-91. 
Troy DJ \& Kerry JP (2010) Consumer perception and the role of science in the meat industry. Meat Science, 86:214-226.

Ubilava D \& Foster K (2009) Quality certification vs. product traceability: Consumer preferences for informational attributes of pork in Georgia. Food Policy, 34:305-310.

Unnevehr L, Eales J, Jensen H, Lusk J, McCluskey J \& Kinsey J (2010) Food and Consumer Economics. American Journal Agricultural Economics, 92:506-521.

Velho JP, Barcellos JOJ, Lengler L, Elias SA \& Oliveira TE (2009) Disposição dos consumidores porto-alegrenses à compra de carne bovina com certificação. Revista Brasileira de Zootecnia, 38:399-404.

Verbeke W \& Vackier I (2004) Profile and effects of consumer involvement in fresh meat. Meat Science, 67:159-168.
Verbeke W, Wezemael LV, Barcellos MD, Kugler JO, Hocquette JF, Ueland O \& Grunert KG (2010) European beef consumers' interest in a beef eating-quality guarantee: insights from a qualitative study in four EU countries. Appetite, 54:289-296.

Wezemael LV, Verbeke W, Kugler JO \& Scholderer J (2011) European consumer acceptance of safety-improving interventions in the beef chain. Food Control, 22:1776-1784.

Zhao R, Qiao J \& Chen Y (2010) Influencing factors of consumer willingness-to-buy traceable foods: An analysis of survey data from two Chinese cities. Agriculture and Agricultural Science Procedia, 1:334-343. 